\title{
Influence of body composition on selected jump performance measures in collegiate female athletes
}

\author{
Christopher J. MacDonald, Michael A. Israetel, Nicole C. Dabbs, Harish Chander, \\ Charles R. Allen, Hugh S. Lamont, John C. Garner
}

Objectives: The monitoring of physical characteristics of athletes is important as it serves to provide valuable information to researchers, coaches, and athletes. The purpose of this work was to identify relationships between body composition and jump performance in collegiate female athletes.

Design: Cross sectional study design with comparisons of relationships.

Methods: Twenty one female collegiate athletes completed testing (Soccer $(n=10)$ : $19.6 \pm 1.3 \mathrm{yrs} ; 165.9 \pm 4.8 \mathrm{~cm} ; 63.7 \pm 8.7 \mathrm{~kg}$; Volleyball $(n=6): 19.8 \pm 1.0 \mathrm{yrs} ; 179.9 \pm 5.1 \mathrm{~cm} ; 76.1 \pm 14.1 \mathrm{~kg}$; and Dance $(n=5): 20.3 \pm 1.8 \mathrm{yrs} ; 163.4 \pm 6.3 \mathrm{~cm} ; 56.8 \pm 6.4 \mathrm{~kg})$. Testing included: dual-energy x-ray absorptiometry (DXA) scans; static jumps (SJ); countermovement jumps (CMJ); and depth jumps (DJ). Data was assessed via Pearson product-moment correlation matrix with two-tailed tests of significance at $\alpha$ level of $\mathrm{p} \leq 0.05$.

Results: Jump modalities related to one another and \%FM and body composition were strong predictors of jump performance. Additionally, jumping metrics reflected the chosen sport participation of the athletes.

Conclusions: Disciplines greater in reliance upon stretch shortening cycle (SSC) utilization (Volleyball) had more and stronger correlations with CMJ and DJ, whereas Soccer and Dance athletes had more and stronger relationships to SJ, as they rely less on a reactive component (than Volleyball) and more on ultimate force production over time.

(Journal of Trainology 2013;2:33-37)

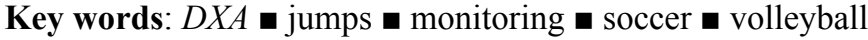

\section{Introduction}

Assessing athletic performance is vital for athletes, coaches, and scientists to identify changes in performance over time and use that information to enhance the training program. Evaluating vertical jumps is a common method used to assess expression of lower body muscular force and power, as well as serving to practically predict performance outcomes. ${ }^{1,2,3}$ Additionally, vertical jump testing is reliable and valid in its estimation of explosive muscular power, ${ }^{4}$ power development, ${ }^{5}$ and as an indicator of performance. ${ }^{6}$

Two primary jumps utilized to measure athletes' jumping ability are the squat or static jump (SJ) and countermovement vertical jump (CMJ). The SJ assesses lower-body concentric strength and power while the CMJ can assess lower-body reactive strength and power (SSC incorporation). ${ }^{7}$ The stretch shortening cycle (SSC) is highly utilized in many sports and sporting activities in which reactive ability is necessary. Furthermore, the more efficient the SSC, the better the rates of force development (RFD), ${ }^{8}$ which itself is an important determinant of athletic performance.

The literature examining the physical characteristics of athletes is abundant. However, the need still exists for data investigating the relationship and predictive nature of anthropometric characteristics to athletic characteristics. Relationships of female athletes' body composition to their athletic performance is especially important as they have a greater propensity for injury (lower limb ligamentous injuries, stress fractures, etc.) compared to their male counterparts, $, 910,11$ with poor body composition and the resultant power-to-weight ratio decrease as a possible culprit. However, this can be obviated by identifying discrepancies and altering the training (if necessary) to benefit the athlete (increased strength, lean body mass and decreased fat mass). ${ }^{12}$ Therefore, the purpose of this work was to determine the relationships between specific measurements of body composition and performance over a variety of vertical jumps in collegiate female athletes. The authors hypothesized that athletes with greater percentage of bone-free lean tissue mass will be better jumpers and that body composition will be a strong predictor of jumping ability. Additionally, athletes with greater reliance on jumping during competition and training are hypothesized to be the better jumpers.

Methods
Participants
Twenty one female athletes, all members of a NCAA DI

Received September 18, 2013; accepted October 24, 2013

From the Department of Kinesiology, Recreation, and Sport Studies, Coastal Carolina University, Conway, SC, USA (C.J.M.), Department of Nutrition and Kinesiology, University of Central Missouri, Warrensburg, MO, USA (M.I.), Department of Kinesiology, California State University, San Bernardino, CA, USA (N.C.D.), Department of Health, Exercise Science and Recreation Management, University of Mississippi, University, MS, USA (H.C., C.R.A., J.C.G.), and Department of Exercise Science, California Lutheran University, Thousand Oaks, CA, USA (H.S.L.) Communicated by Takashi Abe, PhD

Correspondence to Dr. Christopher J. MacDonald, Department of Kinesiology, Recreation, and Sport Studies, Coastal Carolina University, PO Box 261954, Conway, SC 29528, USA. Email: cmacdonal@coastal.edu 
soccer, volleyball, or dance team, volunteered for this testing protocol (all participants $(n=21)=19.8 \pm 1.3 \mathrm{yrs} ; 168.4 \pm 7.5$ $\mathrm{cm} ; 65.5 \pm 12.2 \mathrm{~kg}$; soccer $(n=10)=19.6 \pm 1.3 \mathrm{yrs} ; 165.9 \pm$ $4.8 \mathrm{~cm} ; 63.7 \pm 8.7 \mathrm{~kg}$; volleyball $(n=6)=19.8 \pm 1.0 \mathrm{yrs}$; $179.9 \pm 5.1 \mathrm{~cm} ; 76.1 \pm 14.1 \mathrm{~kg}$; and dance $(n=5)=20.3 \pm 1.8$ yrs; $163.4 \pm 6.3 \mathrm{~cm} ; 56.8 \pm 6.4 \mathrm{~kg}$ ). Athletes were injury free and cleared for participation by their respective coaching staffs. Participants signed University approved Institutional Review Board consent documents (IRB Protocol \# 10-045).

\section{Testing Procedures}

Testing consisted of two sessions, separated by at least 48 hours, during which the athletes were assessed on several measures of anthropometrics and vertical jumps. The initial testing session required measures of standing height (SECA stadiometer, Birmingham, United Kingdom), body mass (SECA digital scale, Birmingham, United Kingdom), and body composition (dual-energy r-ray absorptiometry (DXA), Hologic Discovery W, Bedford, MA). Full body DXA scans were executed with participant supine and measures included total body percentage of fat mass $(\% \mathrm{FM})$; bone mineral content (BMC; g); bone-free lean tissue mass (LTM; kg); and percentage fat free mass (\%FFM; $\mathrm{kg}$ ). The second testing session included all jump protocols. Initial normalized warm up consisting of 5 minutes pedaling a cycle ergometer (Monark cycle ergometer, Vansbro, Sweden) at $0.5 \mathrm{kp}$ between $50-60 \mathrm{rpm}$. After 5 minutes of rest, participants then completed three different jump modalities in the following order: fixed static jump (SJ); fixed countermovement vertical jump (CMJ); and a $30 \mathrm{~cm}$ depth jump (DJ) with a $50 \%$ effort jump preceding each condition. One minute rest was allotted between jumps and 3 minutes between jump types. The SJ was performed from a $90^{\circ}$ knee angle with participants maximally jumping vertically on cue, with no countermovement. SJ characteristics were: jump height (SJ $\mathrm{Ht}(\mathrm{cm})$ ); force per $\mathrm{kg}$ body mass (SJ Force/kg (N/kg)); power per kg body mass (SJ Power/kg (Watts/kg)); and velocity (SJ Peak Vel $(\mathrm{cm} / \mathrm{s}))$. For the CMJ, athletes descending to a selfselected depth and jumped as high as possible. CMJ measures included: jump height (CMJ $\mathrm{Ht}(\mathrm{cm})$ ); force per $\mathrm{kg}$ body mass (CMJ Force/kg (N/kg)); power per kg body mass (CMJ Power/ $\mathrm{kg}$ (Watts $/ \mathrm{kg})$ ); and velocity (CMJ Peak Vel $(\mathrm{cm} / \mathrm{s}))$. The DJ was executed with athletes standing on a $30 \mathrm{~cm}$ box, stepping off and upon landing, attempting to jump vertically as high as possible while (verbal command was given to) minimizing ground contact time. DJ measures included: force (DJ Force (N)); power (DJ Power (Watts)); and velocity (DJ Peak Vel $(\mathrm{cm} / \mathrm{s}))$. For all jumps, hand position was fixed just superior to the lateral iliac crest.

All jump data was collected with a MyoTest ${ }^{\circledR}$ (MyoTest SA, Sion, Switzerland) triaxial accelerometer device at preset rates suggested by the equipment manufacturer (SJ and CMJ at 200 $\mathrm{Hz}$, DJ at $500 \mathrm{~Hz}$ ). The MyoTest ${ }^{\circledR}$ was placed on an adjustable belt, just below the fixed location of the hands on the left side, for all jumps and data was averaged from 3 maximal trials of each jumping condition.

\section{Statistical Analysis}

A Pearson product-moment correlation matrix with twotailed tests of significance were performed (a priori alpha level of $\mathrm{p} \leq 0.05$ ) on all data using PASW Statistics 18 . Correlational strengths were based on the following ranges: $0.0-0.1$ is trivial or very small; $0.1-0.3$ is small, low, or minor; $0.3-0.5$ is moderate or medium; $0.5-0.7$ is large, high, or major; $0.7-0.9$ is very large or very high; and $0.9-$ 1.0 is nearly perfect to perfect. ${ }^{13,14}$

Table 1 Statistically significant $(p \leq 0.05)$ relationships of SJ data considering All Participants, Soccer only, Dance only, and Volleyball only. Data is ordered from stronger to weaker $\boldsymbol{r}$ value, within each group.

\begin{tabular}{|c|c|c|}
\hline \multicolumn{3}{|c|}{ SJ (mean \pm standard deviation) } \\
\hline \multicolumn{3}{|c|}{ All Participants } \\
\hline SJ Power $/ \mathrm{kg}(40.90 \pm 6.65)$ & SJ Peak Vel $(220.24 \pm 23.17)$ & 0.91 \\
\hline SJ Force/kg $(24.27 \pm 2.91)$ & SJ Power $/ \mathrm{kg}(40.90 \pm 6.65)$ & 0.81 \\
\hline SJ Ht $(27.42 \pm 5.04)$ & CMJ Ht $(31.72 \pm 5.52)$ & 0.77 \\
\hline SJ Power $/ \mathrm{kg}(40.90 \pm 6.65)$ & DJ Peak Vel $(264.05 \pm 28.91)$ & 0.65 \\
\hline SJ Ht $(27.42 \pm 5.04)$ & CMJ Peak Vel $(214.67 \pm 39.99)$ & 0.61 \\
\hline SJ Ht $(27.42 \pm 5.04)$ & DJ Peak Vel $(264.05 \pm 28.91)$ & 0.59 \\
\hline SJ Ht $(27.42 \pm 5.04)$ & SJ Peak Vel $(220.24 \pm 23.17)$ & 0.59 \\
\hline SJ Ht $(27.42 \pm 5.04)$ & $\%$ FM $(22.91 \pm 4.89)$ & -0.58 \\
\hline SJ Force/kg $(24.27 \pm 2.91)$ & DJ Peak Vel $(264.05 \pm 28.91)$ & 0.57 \\
\hline SJ Ht $(27.42 \pm 5.04)$ & CMJ Power $/ \mathrm{kg}(40.45 \pm 9.77)$ & 0.57 \\
\hline SJ Power $/ \mathrm{kg}(40.90 \pm 6.65)$ & CMJ Ht $(31.72 \pm 5.52)$ & 0.56 \\
\hline SJ Force/kg $(24.27 \pm 2.91)$ & SJ Peak Vel $(220.24 \pm 23.17)$ & 0.55 \\
\hline SJ Peak Vel $(220.24 \pm 23.17)$ & DJ Peak Vel $(264.05 \pm 28.91)$ & 0.54 \\
\hline SJ Peak Vel $(220.24 \pm 23.17)$ & CMJ Ht $(31.72 \pm 5.52)$ & 0.53 \\
\hline SJ Ht $(27.42 \pm 5.04)$ & SJ Power $/ \mathrm{kg}(40.90 \pm 6.65)$ & 0.47 \\
\hline SJ Peak Vel $(220.24 \pm 23.17)$ & DJ Power $(3919.05 \pm 851.64)$ & 0.47 \\
\hline SJ Power $/ \mathrm{kg}(40.90 \pm 6.65)$ & DJ Power $(3919.05 \pm 851.64)$ & 0.46 \\
\hline SJ Ht $(27.42 \pm 5.04)$ & DJ Power (3919.05 \pm 851.64$)$ & 0.45 \\
\hline SJ Power/kg $(40.90 \pm 6.65)$ & CMJ Power $/ \mathrm{kg}(40.45 \pm 9.77)$ & 0.44 \\
\hline \multicolumn{3}{|c|}{ Soccer } \\
\hline SJ Power $/ \mathrm{kg}(38.38 \pm 8.08)$ & SJ Peak Vel $(213.10 \pm 27.77)$ & 0.97 \\
\hline SJ Force/kg $(23.04 \pm 2.88)$ & SJ Power $/ \mathrm{kg}(38.38 \pm 8.08)$ & 0.93 \\
\hline SJ Force/kg $(23.04 \pm 2.88)$ & SJ Peak Vel $(213.10 \pm 27.77)$ & 0.86 \\
\hline SJ Ht $(26.09 \pm 4.93)$ & CMJ Ht $(29.46 \pm 4.37)$ & 0.81 \\
\hline SJ Ht $(26.09 \pm 4.93)$ & $\% \mathrm{FM}(21.77 \pm 6.05)$ & -0.78 \\
\hline SJ Ht $(26.09 \pm 4.93)$ & DJ Peak Vel $(252.00 \pm 28.00)$ & 0.72 \\
\hline SJ Force $/ \mathrm{kg}(23.04 \pm 2.88)$ & DJ Power $(3632.00 \pm 641.97)$ & 0.71 \\
\hline SJ Power/kg (38.38 \pm 8.08$)$ & CMJ Ht $(29.46 \pm 4.37)$ & 0.70 \\
\hline SJ Peak Vel $(213.10 \pm 27.77)$ & CMJ Ht $(29.46 \pm 4.37)$ & 0.70 \\
\hline SJ Power $/ \mathrm{kg}(38.38 \pm 8.08)$ & DJ Peak Vel $(252.00 \pm 28.00)$ & 0.64 \\
\hline SJ Peak Vel $(213.10 \pm 27.77)$ & DJ Peak Vel $(252.00 \pm 28.00)$ & 0.63 \\
\hline \multicolumn{3}{|c|}{ Dance } \\
\hline SJ Force/kg $(25.14 \pm 2.24)$ & CMJ Force $/ \mathrm{kg}$ & 0.95 \\
\hline SJ Peak Vel $(229.40 \pm 10.26)$ & LTM $(40.81 \pm 4.34)$ & 0.94 \\
\hline SJ Peak Vel $(229.40 \pm 10.26)$ & BMC $(2346.74 \pm 210.78)$ & 0.94 \\
\hline SJ Peak Vel $(229.40 \pm 10.26)$ & $\mathrm{BM}(56.79 \pm 6.42)$ & 0.94 \\
\hline SJ Ht $(28.70 \pm 3.04)$ & SJ Peak Vel $(229.40 \pm 10.26)$ & 0.90 \\
\hline SJ Force/kg $(25.14 \pm 2.24)$ & SJ Power $/ \mathrm{kg}(43.72 \pm 3.72)$ & 0.88 \\
\hline \multicolumn{3}{|c|}{ Volleyball } \\
\hline SJ Ht $(29.50 \pm 6.50)$ & SJ Peak Vel $(226.14 \pm 20.22)$ & 0.91 \\
\hline SJ Ht $(29.50 \pm 6.50)$ & CMJ Peak Vel $(236.00 \pm 32.40)$ & 0.82 \\
\hline
\end{tabular}




\section{Results}

The results from the correlation analyses are presented as only the statistically significant relationships (in descending order of strength) between the measures of anthropometrics, SJ (Table 1), CMJ (Table 2), and DJ (Table 3) and subclassified as All Participants, Soccer only, Dance only, or Volleyball only.

Table 2 Statistically significant $(p \leq 0.05)$ relationships of CMJ data considering All Participants, Soccer only, Dance only, and Volleyball only. Data is ordered from stronger to weaker $\boldsymbol{r}$ value, within each group.

\begin{tabular}{|c|c|c|}
\hline \multicolumn{3}{|c|}{ CMJ (mean \pm standard deviation) } \\
\hline \multicolumn{3}{|c|}{ All Participants } \\
\hline CMJ Power $/ \mathrm{kg}(40.45 \pm 9.77)$ & CMJ Peak Vel $(214.67 \pm 39.99)$ & 0.97 \\
\hline CMJ Force/kg (23.14 \pm 2.05$)$ & CMJ Power $/ \mathrm{kg}(40.45 \pm 9.77)$ & 0.77 \\
\hline CMJ Ht (31.72 \pm 5.52$)$ & CMJ Power/kg $(40.45 \pm 9.77)$ & \\
\hline CMJ Ht (31. & CMJ Peak Vel (214.67 \pm 39.99$)$ & 0.74 \\
\hline CMJ Ht (31.72 \pm 5.52$)$ & $5 \pm 28.91)$ & \\
\hline CMJ Force $/ \mathrm{kg}(23.14 \pm 2.05)$ & $\mathrm{CM}$ & 0.70 \\
\hline CMJ Power $/ \mathrm{kg}(40.45 \pm 9.77)$ & DJ Peak Vel $(264.05 \pm 28$ & \\
\hline CMJ Force/kg $(23.14 \pm 2.05)$ & $\%$ FM $(22.9$ & \\
\hline CMJ Ht $(31.72 \pm 5.52)$ & $\%$ FM $(22.91$ & -0.64 \\
\hline CMJ Peak Vel $(214.67 \pm 39.99)$ & DJ Peak Vel (264.05 & \\
\hline CMJ Force/kg $(23.14 \pm 2.05)$ & DJ Peak Vel $(264.05 \pm 28$ & 0. \\
\hline CMJ Ht $(31.72 \pm 5.52)$ & $\mathrm{CMJ}$ & \\
\hline CMJ Power $/ \mathrm{kg}(40.45 \pm 9.77)$ & $\% \mathrm{FM}$ & -0.55 \\
\hline CMJ Peak Vel $(214.67 \pm 39.99)$ & $\% \mathrm{FM}$ & -0.55 \\
\hline CMJ Ht $(31.72 \pm 5.52)$ & DJ Po & \\
\hline CMJ Force/kg (23.14 \pm 2.05$)$ & DJ Pc & 0.51 \\
\hline CMJ Power $/ \mathrm{kg}(40.45 \pm 9.77)$ & DJ F & \\
\hline \multicolumn{3}{|c|}{ Soccer } \\
\hline CMJ Power $/ \mathrm{kg}(37.38 \pm 8.49)$ & CMJ Pea & 0.94 \\
\hline CMJ Ht $(29.46 \pm 4.37)$ & DJ P & \\
\hline CMJ Force/kg $(22.80 \pm 2.26)$ & $38 \pm 8$ & 0.73 \\
\hline CMJ Force/kg $(22.80 \pm 2.26)$ & $\mathrm{CMJ}$ & \\
\hline CMJ Power $/ \mathrm{kg}(37.38 \pm 8.49)$ & DJ P & 0.69 \\
\hline CMJ Peak Vel $(200.20 \pm 35.67)$ & DJ Pea & 0.67 \\
\hline \multicolumn{3}{|c|}{ Dance } \\
\hline $42.14 \pm 11.63)$ & CMJ Pea & 0.99 \\
\hline \multicolumn{3}{|c|}{ Volleyball } \\
\hline $45.27 \pm 9.82)$ & CMJ Peak & 0.99 \\
\hline \pm 6.81$)$ & $\mathrm{CMJ}$ & 0.94 \\
\hline \pm 6.81$)$ & $236.00 \pm 32.40)$ & 0.92 \\
\hline $\mathrm{e} / \mathrm{kg}(23.84 \pm 2.05)$ & wer $/ \mathrm{kg}(45.27 \pm$ & 0.88 \\
\hline CMJ Power $/ \mathrm{kg}(45.27 \pm 9.82)$ & $\% \mathrm{FM}(22.30 \pm 5.81)$ & -0.87 \\
\hline CMJ Force/kg (23.84 \pm 2.05$)$ & $\% \mathrm{FM}$ & -0.86 \\
\hline CMJ Force/kg $(23.84 \pm 2.05)$ & CMJ Peak Vel $(236.00 \pm$ & 0.84 \\
\hline CMJ Power $/ \mathrm{kg}(45.27 \pm 9.82)$ & DJ Peak Vel & 0.84 \\
\hline CMJ Force/kg (23.84 \pm 2.05$)$ & DJ Peak Vel $(270.43 \pm 29.74)$ & 0.83 \\
\hline CMJ Peak Vel $(236.00 \pm 32.40)$ & $\% \mathrm{FM}(22.30 \pm 5.81)$ & -0.83 \\
\hline CMJ Ht $(35.84 \pm 6.81)$ & $\% \mathrm{FM}(22.30 \pm 5.81)$ & -0.82 \\
\hline CMJ Ht $(35.84 \pm 6.81)$ & DJ Peak Vel $(270.43 \pm 29.74)$ & 0.82 \\
\hline CMJ Ht $(35.84 \pm 6.81)$ & CMJ Force $/ \mathrm{kg}(23.84 \pm 2.05)$ & 0.80 \\
\hline CMJ Peak Vel $(236.00 \pm 32.40)$ & DJ Peak Vel $(270.43 \pm 29.74)$ & 0.80 \\
\hline
\end{tabular}

Table 3 Statistically significant $(p \leq 0.05)$ relationships of DJ data considering All Participants, Soccer only, and Volleyball only. Data is ordered from stronger to weaker $r$ value, within each group.

\begin{tabular}{|c|c|c|}
\hline \multicolumn{3}{|c|}{ DJ (mean \pm standard deviation) } \\
\hline \multicolumn{3}{|c|}{ All Participants } \\
\hline DJ Force $(3113.33 \pm 648.96)$ & BMC $(2729.49 \pm 353.13)$ & 0.69 \\
\hline DJ Power $(3919.05 \pm 851.64)$ & LTM $(48.31 \pm 7.70)$ & 0.69 \\
\hline DJ Power $(3919.05 \pm 851.64)$ & $\%$ FFM $(77.09 \pm 4.89)$ & 0.69 \\
\hline DJ Force $(3113.33 \pm 648.96)$ & $\%$ FFM $(77.09 \pm 4.89)$ & 0.61 \\
\hline DJ Force $(3113.33 \pm 648.96)$ & LTM $(48.31 \pm 7.70)$ & 0.61 \\
\hline DJ Power $(3919.05 \pm 851.64)$ & DJ Peak Vel $(264.05 \pm 28.91)$ & 0.61 \\
\hline DJ Peak Vel $(264.05 \pm 28.91)$ & $\% \mathrm{FM}(22.91 \pm 4.89)$ & -0.58 \\
\hline DJ Force $(3113.33 \pm 648.96)$ & BMC $(2729.49 \pm 353.13)$ & 0.58 \\
\hline DJ Power $(3919.05 \pm 851.64)$ & BMC $(2729.49 \pm 353.13)$ & 0.55 \\
\hline DJ Power $(3919.05 \pm 851.64)$ & $\mathrm{BM}(67.63 \pm 11.92)$ & 0.47 \\
\hline DJ Force $(3113.33 \pm 648.96)$ & DJ Power $(3919.05 \pm \varepsilon$ & 0.46 \\
\hline \multicolumn{3}{|c|}{ Soccer } \\
\hline DJ Force $(3306.00 \pm 664.83)$ & $\mathrm{BM}(63.75 \pm 8.71)$ & 0.71 \\
\hline DJ Power $(3632.00 \pm 641.97)$ & DJ Peak Vel $(252.00 \pm 28.00$ & 0.65 \\
\hline DJ Force $(3306.00 \pm 664.83)$ & LTM $(45.99 \pm 3.86)$ & 0.65 \\
\hline DJ Force $(3306.00 \pm 664.83)$ & $\%$ FFM $(78.23 \pm 6.05)$ & 0.65 \\
\hline \multicolumn{3}{|c|}{ Volleyball } \\
\hline DJ Force $(3320.00 \pm 616.28)$ & BMC $(3095.50 \pm 391.11)$ & 0.87 \\
\hline DJ Peak Vel $(270.43 \pm 29.74)$ & $\% \mathrm{FM}(22.30 \pm 5.81)$ & -0.79 \\
\hline DJ Force $(3320.00 \pm 616.28)$ & $\mathrm{BM}(76.09 \pm 14.08)$ & 0.78 \\
\hline DJ Force $(3320.00 \pm 616.28)$ & SJ Force $/ \mathrm{kg}(25.27 \pm 2$ & 0.77 \\
\hline DJ Force $(3320.00 \pm 616.28)$ & $\%$ FFM $(77.70 \pm 5.81)$ & 0.76 \\
\hline
\end{tabular}

\section{Anthropometrics}

Data indicated that all body composition measures, especially those related to $\% \mathrm{FM}$ and $\% \mathrm{FFM}$, are closely associated.

\section{SJ}

Table 1 illustrates the relationships of SJ to the other jumps and to body composition measures. The SJ showed moderate to strong correlations to all jumping conditions for All Participants $(r=0.44$ through 0.91$)$, Soccer $(r=0.63$ through $0.97)$, Volleyball $(r=0.82$ through 0.91$)$, and Dance $(r=0.88$ through 0.95). Dance also showed strong relationships between the SJ measures and BM $(r=0.94)$, BMC $(r=0.94)$, and LTM $(r=0.94)$, while strong inverse relationships were identified between SJ and \%FM for All Participants $(r=-0.58)$ and for Soccer $(r=-.078)$.

\section{CMJ}

Table 2 indicates the relationships of CMJ to the other jumps and to body composition measures. The CMJ showed moderate to strong correlations to all jumping conditions for All Participants $(r=0.44$ through 0.97$)$, Soccer $(r=0.67$ through 0.94$)$, Volleyball $(r=0.80$ through 0.99$)$, and Dance $(r$ $=0.99$ ) while, in addition, there were strong inverse relationships for the $\mathrm{CMJ}$ and \%FM for All Participants $(\mathrm{r}=$ -0.55 through -0.66$)$ and for Volleyball $(r=-.082$ through $-0.87)$. 


\section{DJ}

Table 3 illustrates the relationships of DJ to the other jumps and to body composition measures. The DJ showed moderate to strong correlations to all jumping conditions for All Participants (0.46 through 0.61), Soccer $(r=0.65)$, and Volleyball $(\mathrm{r}=0.77)$. There were moderate to strong relationships between DJ and BMC, \%FFM, BM and LTM for All Participants, Soccer, and Volleyball $(r=0.47$ through 0.87) while strong inverse relationships existed between the DJ and $\%$ FM for All Participants $(\mathrm{r}=-0.58)$ and for Volleyball $(\mathrm{r}=$ $-0.79)$.

\section{Discussion}

It is expected that measures of jumping performance be predictive of one another as they are ultimately measures of muscular force production and similar patterns of expression from the same lower extremities. ${ }^{15,16}$ The predictive nature of body composition, however, is noteworthy. The data shows the particular relationships between $\% \mathrm{FM}$ of the athletes and jumping performance. It is clearly demonstrated that a strong predictive factor of better jumping (and theoretically, improved athletic performance) is decreased \%FM. This indicates that ultimately improving body composition is associated with (or can be a predictor of) better jumping performance. If improved body composition is directly related to better performance, then training athletes to increase LTM (by including hypertrophic protocols and exercises at various times during the training year) and reduce fat mass should be paramount when considering training and nutritional schemes. Additionally, the reviewed literature reports successful methods for training athletes to specifically achieve increases in hypertrophy, muscular strength, power, or jumping performance and these protocols also ultimately increased athletic performance. ${ }^{17,18,19}$

The strong inverse relationships between SJ and \%FM for All Participants and for Soccer as well as the strong relationships between the $\mathrm{SJ}$ measures and $\mathrm{BM}, \mathrm{BMC}$, and LTM for Dance provide an important conclusion. This data illustrates that the particular requirements of these disciplines (i.e. greater time course of force generation, when compared to Volleyball) have a very specific carry over to the outcome measures assessed, in this case the SJ.

The CMJ showed moderate to strong correlations for all jumping conditions for All Participants and strong inverse relationships between the $\mathrm{CMJ}$ and \%FM for All Participants and Volleyball. This data, as did the SJ data, illustrates that the particular requirements of a sporting disciplines carry over to the closely related outcome measure. That is, the CMJ, an activity that fulfills a large percentage of the sporting requirement of Volleyball, especially when compared to Soccer and Dance, has strong relationships to the body composition of these athletes ${ }^{20}$ while movement pattern specificity of the CMJ is also very similar to Volleyball (high force, high velocity, large involvement of the SSC, reactive jumping) especially when compared to Soccer and Dance.

The DJ showed moderate to strong correlations to all jumping conditions for All Participants, Soccer, and Volleyball as well as strong inverse relationships between the DJ and $\%$ FM for both All Participants and Volleyball. Additionally, strong relationships between DJ and measures BMC, \%FFM, BM and LTM were identified for All Participants, Soccer, and Volleyball. The DJ not having any statistically significant relationship to Dance is likely because of the nature of the training and competition activities of the Dance group seldom requiring a reactive component. That is, the requirements of Soccer and Volleyball may include explosive movements such as jumping/landing/cutting/running that can be immediately followed by another explosive movement and this reactive nature of training and competition is manifested by DJ ability.

\section{Conclusion}

In conclusion, athletic/physiological characteristics must be monitored in order to better understand the training process of athletes, identify depressed performance, and to assess the long term progress of individuals and teams. This work illustrated relationships in some of the more commonly measured characteristics, especially at the beginning of a long term monitoring program. From this data, the authors identify two major conclusions: 1) outside of jump modalities relating to one another, $\% \mathrm{FM}$ and ultimately body composition are important predictors of jumping performance and; 2) jumping metrics can and will reflect the athletes that they measure. The athletes who compete and train with more reactive, explosive, high velocity countermovement activities (Volleyball) will have more significant, stronger correlations with the CMJ and DJ. ${ }^{20}$ The athletes with who compete and train with a combination of the explosive activities and those intended to maximize force production (Soccer and Dance) will result in stronger significant relationships with SJ, while still producing some relationships with CMJ and DJ. ${ }^{21}$ Future research should include evaluation of the athletes' strength levels, where the athletes are in their training programs/competition year, and their overall training status.

\section{Acknowledgements}

The authors of this work would like to thank all of the athletes, coaches, administrators, and researchers who gave their time and resources to see this project through.

\section{References}

1. Arendt E, Dick R. Knee injury patterns among men and women in collegiate basketball and soccer. NCAA data and review of literature. Am J Sport Med 1995; 23: 694-701.

2. Hewett TE, Lindenfeld TN, Riccobene JV, et al. The effect of neuromuscular training on the incidence of knee injury in female athletes. Am J Sport Med 1999; 27: 699-706.

3. Markovic G, Dizdar D, Jukic I, et al. Reliability and factorial validity of squat and countermovement jump tests. J Strength Cond Res 2004; 18: 551-555.

4. Bobbert MF, Casius LJR. If the effect of a countermovement on jump height due to active state development? Med Sci Sport Exer 2005; 37: 440-446.

5. Ferretti A, Papandrea P, Conteduca F, et al. Knee ligament injuries in volleyball players. Am J Sport Med 1992; 20: 203-207.

6. Flanagan EP, Comyns TM. The use of contact time and the reactive strength index to optimize fast stretch-shortening cycle training. Strength 
Cond J 2008; 30: 32-38.

7. Stone MH, Plisk SS, Stone ME, et al. Athletic performance development: volume load - 1 set vs. multiple sets, training velocity and training variation. Strength Cond 1998; 20: 22-31.

8. Walshe AD, Wilson GJ, Murphy AJ. The validity and reliability of a test of lower body musculotendinous stiffness. Eur J Appl Physiol 1996; 73: 332339.

9. Hosler WW, Morrow JR, Jackson AS. Strength, anthropometric and speed characteristic of college women volleyball players. Res Quart 1978; 49: 385-388.

10. Kraemer WJ, Ramatess NA. Fundamentals of resistance training: progression and exercise prescription. Med Sci Sport Exer 2004; 36: 674688.

11. Kraemer WJ, Stone MH, O'Bryant H, et al. Effects of single versus multiple sets of weight training: impact of volume, intensity and variation. J Strength Cond Res 1997; 11: 143-147.

12. Wilson G, Murphy A. The efficacy of isokinetic, isometric and vertical jump tests in exercise science. Australian J Sci Med Sport 1995; 27: 20-24.

13. Hopkins WG. Measures of reliability in sports medicine and science. Sports Med 2000; 30: 1-15.

14. Hopkins WG, Hawley JA, Burke LM. Design and analysis of research on sport performance enhancement. Med Sci Sport Exer 1999; 31: 472-485.

15. Bosco C, Mognoni P, Luhtanen P. Relationship between isokinetic performance and ballistic movement. Eur J Appl Physiol 1983; 51: 357364.

16. Ravn S, Voigt M, Simonsen EB, et al. Choice of jumping strategy in two standard jumps, squat and countermovement jump - effect of training background or inherited preference? Scand J Med Sci Spor 1999; 9: 201208.

17. Bobbert MF, Gerritsen KG, Litjens MC, et al. Why is countermovement jump height greater than squat jump? Med Sci Sport Exer 1996; 28: $1402-$ 1412.

18. Cormie P, McBride JM, McCaulley GO. Power-time, force-time, and velocity-time curve analysis of the countermovement jump: impact of training. J Strength Cond Res 2009; 23: 177-186.

19. Stone MH, O'Bryant H, Garhammer J. A hypothetical model for strength training. J Sport Med Phys Fit 1981; 21: 342-351.

20. Fleck SJ, Case S, Puhl J, et al. Physical and physiological characteristics of elite women volleyball players. Canadian J Appl Sport Sci 1985; 10: 122126.

21. Stolen T, Chamari K, Castagna C, et al. Physiology of soccer: an update. Sports Med 2005; 35: 501-536. 